Submitted to ApJ OCtober 31, 2012; ACCepted June 7, 2013

Preprint typeset using $\mathrm{IAT}_{\mathrm{E}} \mathrm{X}$ style emulateapj v. 5/2/11

\title{
A DETAILED KINEMATIC MAP OF CASSIOPEIA A'S OPTICAL MAIN SHELL AND OUTER HIGH-VELOCITY EJECTA
}

\author{
Dan Milisavljevic ${ }^{1}$ and Robert A. Fesen ${ }^{2}$ \\ ${ }^{1}$ Harvard-Smithsonian Center for Astrophysics, 60 Garden Street, Cambridge, MA, 02138 \\ email: dmilisav@cfa.harvard.edu \\ ${ }^{2} 6127$ Wilder Lab, Department of Physics \& Astronomy, Dartmouth College, Hanover, NH 03755 \\ Submitted to ApJ October 31, 2012; accepted June 7, 2013
}

\begin{abstract}
We present three-dimensional kinematic reconstructions of optically emitting material in the young Galactic supernova remnant Cassiopeia A (Cas A). These Doppler maps have the highest spectral and spatial resolutions of any previous survey of Cas A and represent the most complete catalog of its optically emitting material to date. We confirm that the bulk of Cas A's optically bright ejecta populate a torus-like geometry tilted approximately $30^{\circ}$ with respect to the plane of the sky with a -4000 to $+6000 \mathrm{~km} \mathrm{~s}^{-1}$ radial velocity asymmetry. Near-tangent viewing angle effects and an inhomogeneous surrounding CSM/ISM environment suggest that this geometry and velocity asymmetry may not be faithfully representative of the remnant's true 3D structure or the kinematic properties of the original explosion. The majority of the optical ejecta are arranged in several well-defined and nearly circular ring-like structures with diameters between approximately $30^{\prime \prime}(0.5 \mathrm{pc})$ and $2^{\prime}(2 \mathrm{pc})$. These ejecta rings appear to be a common phenomenon of young core-collapse remnants and may be associated with post-explosion input of energy from plumes of radioactive ${ }^{56} \mathrm{Ni}$-rich ejecta that rise, expand, and compress non-radioactive material. Our optical survey also encompassed Cas A's faint outlying ejecta knots and exceptionally high-velocity NE and SW streams of S-rich debris often referred to as 'jets'. These outer knots, which exhibit a chemical make-up suggestive of an origin deep within the progenitor star, appear to be arranged in opposing and wide-angle outflows with opening half-angles of $\approx 40^{\circ}$.

Subject headings: ISM: supernova remnants - ISM: individual objects (Cassiopeia A) — supernovae: general
\end{abstract}

\section{INTRODUCTION}

A variety of observations and hydrodynamic modeling make a compelling case that high-mass, core-collapse supernovae (SNe) are intrinsically aspherical events with highly clumped ejecta caused by dynamical instabilities (Wang \& Wheeler 2008; Maeda et al. 2008; Nordhaus et al. 2010; Janka 2012; Tanaka et al. 2012). The origin of the expansion asphericities is currently uncertain. Possible causes include asymmetrical neutrino heating and accretion-shock instabilities (Kifonidis et al. 2000; Blondin et al. 2003; Kifonidis et al. 2003; Burrows et al. 2006; Scheck et al. 2006; Marek \& Janka 2009; Hanke et al. 2012), and the influences of rotation and magnetic fields (Wheeler et al. 2002; Akivama et al. 2003; Frver \& Warren 2004; Shibata et al. 2006; Masada et al. 2012).

Observations of the kinematic and chemical properties of SN ejecta can help investigate which of the aforementioned explosion mechanisms may dominate. For example, late-time optical spectra obtained $t \gtrsim 6$ months beyond outburst in strippedenvelope events often exhibit multi-peaked emission line profiles consistent with explosion models simulating aspherical axisymmetric and potentially jetrelated explosions viewed along different angles from the equatorial plane (Mazzali et al. 2005; Modiaz et al. 2008; Maeda et al. 2008; Taubenberger et al. 2009; Milisavljevic et al. 2010). Additional clues for constraining explosion mechanisms come from spectropolarimetry. These studies show that ejecta can be asymmet- ric in the inner layers, supporting the view that the explosion process is strongly aspherical (Wang \& Wheeler 2008; Tanaka et al. 2012).

An alternative way to investigate core-collapse explosion asymmetries is through observations of young Galactic supernova remnants (SNRs). Studies of the handful of young O-rich Galactic remnants believed to be the results of massive stars allow one to probe kinematic asymmetries in the expanding ejecta at spatial scales not possible from extragalactic SN observations. They can also offer clues about the explosive mixing of chemically distinct zones in the progenitor star and the nature of the central compact remnant.

The young Galactic remnant Cassiopeia A (Cas A) provides perhaps the clearest look at the explosion dynamics of a high mass SN. With an explosion date most likely around $1681 \pm 19$, Cas $\mathrm{A}$ is the youngest Galactic core-collapse SNR known (Thorstensen et al. 2001; Fesen et al. 2006b). At an estimated distance of $3.4 \mathrm{kpc}$ (Reed et al. 1995), it is also among the closest.

Cas A is the only historical core-collapse SNR with a secure SN subtype classification. The detection of light echoes of the supernova outburst (Rest et al. 2008, 2011; Besel \& Krause 2012) has enabled follow-up optical spectral observations which indicate that the original supernova associated with Cas A exhibited an optical spectrum at maximum light similar to those seen for the Type IIb events SN 1993J and SN 2003bg (Krause et al. 2008; Rest et al. 2011).

Cas $\mathrm{A}$ is inferred to have undergone extensive mass 
loss from its original $20-25 \mathrm{M}_{\odot}$ progenitor to only $3-4 \mathrm{M}_{\odot}$ upon explosion, leaving a relatively dense and slow moving remnant stellar wind (Chevalier \& Oishi 2003). Such conditions do not generally arise with a radiatively driven wind from a $20-25 \mathrm{M}_{\odot}$ progenitor, but require the existence of a binary companion to aid the mass loss (Woosley et al. 1993; Young et al. 2006; Claeys et al. 2011).

Several Doppler reconstructions of Cas A's main shell ejecta have been conducted in the past using optical, infrared, and X-ray data. Results of these studies have revealed significant ejecta asymmetries potentially related to the explosion dynamics. For example, optical studies have shown that much of the remnant's ejecta are arranged in large rings on a spherical shell exhibiting an overall velocity asymmetry of -4000 to $+6000 \mathrm{~km} \mathrm{~s}^{-1}$ (Minkowski 1959; Lawrence et al. 1995; Reed et al. 1995). Spectra of surrounding light echos also indicate strong asymmetry in the Cas A supernova's photosphere (Rest et al. 2011).

The recent study of the Cas A remnant by DeLaney et al. (2010) presented an extensive multiwavelength Doppler reconstruction using new velocity measurements from Spitzer infrared and Chandra Xray observations combined with previous optical data on the remnant's highest velocity, outer ejecta from Fesen (2001). Besides confirming the presence of several large ejecta rings in the infrared, they interpreted the structure of Cas A's bright main shell of ejecta knots to consist of a spherical component, a tilted thick disk, multiple ejecta jets/pistons, and optical fast-moving knots all populating the thick disk plane. They concluded that the bulk of the symmetries and asymmetries seen in Cas A are properties intrinsic to the supernova explosion.

One often cited piece of observational evidence that the Cas A progenitor underwent a highly aspherical explosion has been the presence of a 'flare' or 'jet' of unusually high-velocity SN debris extending some $300^{\prime \prime}$ out along the northeast (NE) limb from the remnant center (about twice that of main shell ejecta) at a position angle of $\sim 70^{\circ}$ and visible even in the earliest photographic images of the remnant (Minkowski 1968; van den Bergh \& Dodd 1970). Ejecta knots in this NE region exhibit proper motions indicating expansion velocities extending up to $14,000(\mathrm{D} / 3.4 \mathrm{kpc}) \mathrm{km} \mathrm{s}^{-1}$, some $8000 \mathrm{~km} \mathrm{~s}^{-1}$ faster than the fastest main shell ejecta knots (Fesen 2001; Fesen et al. 2006b).

A fainter and considerably sparser southwest (SW) so-called 'counterjet' of equally high-velocity ejecta was discovered optically (Fesen 2001) and subsequently confirmed in X-rays and the infrared (Hwang et al. 2004; Hines et al. 2004). Chandra X-ray images show the SW jet to be much fainter and not as extended radially as the NE one, and in the optical only about 200 knots have been identified compared to over 1000 in the NE jet. One reason for this substantial difference, at least in the optical, may lie in greater line of sight extinction $\left(\mathrm{A}_{V} \approx 5-8 \mathrm{mag}\right)$ suspected along the remnant's western boundary (Keohane et al. 1996; Willingale et al. 2002).

Some theoretical models have suggested that bipolar jets may be associated with the explosion dynamics. For example, numerical simulations of MHD jet and neutrino-driven expansion models produce aspher- ical jets with ejection velocities and axial expansion ratios $\sim 2$ not unlike those seen for Cas A (Khokhlov et al. 1999; Kotake et al. 2005). While an X-ray analysis of Cas A's NE and SW jets concluded that they are unlikely to have played an important role in the explosion mechanism (Laming et al. 2006), the kinematic properties of the NE and SW jets have not been well determined and the limited data currently available for these regions cannot constrain whether or not the two streams even comprise a genuine bipolar, jet-counterjet expansion structure. Such information is crucial to assess their potential relationship to core-collapse explosion dynamics.

The need for an in-depth study of the kinematic properties of the NE and SW ejecta jets and their relation to the remnant's main shell of reverse shock heated ejecta motivated us to undertake a deep reconnaissance of the entire Cas A remnant with high spatial and spectral resolution. In Sections 2 and 3 we describe the observations and our methods for reducing the data. This is followed with a brief description of how these data were used to develop a three dimensional (3D) Doppler reconstruction of the optically emitting ejecta in Section 4. In Sections [5] and 6] the kinematic properties of the ejecta are presented and discussed. We summarize our findings and discuss potential future work in Section 7

\section{OBSERVATIONS}

A series of observing runs starting September 2007 and continuing to November 2010 were conducted to obtain low-dispersion long-slit optical spectra across the entire remnant. Most observations were carried out at MDM Observatory, on Kitt Peak, AZ using the $2.4 \mathrm{~m}$ Hiltner telescope. These observations were later supplemented by both multi-slit and long-slit spectra obtained with the Kitt Peak Mayall 4m telescope. A summary of all observations is given in Table 1. Additional relevant details of these observations are discussed below. A finding chart of all slit positions is presented in Figure 1 .

For Cas A's main shell ejecta, the MDM Modular Spectrograph (Modspec) with a SITe $2048 \times 2048$ CCD detector ('Echelle') on the MDM $2.4 \mathrm{~m}$ telescope was used. A $2^{\prime \prime} \times 5^{\prime}$ long-slit oriented north-south was spaced every $3^{\prime \prime}$. Exposures were generally $2 \times 500 \mathrm{~s}$. The effective wavelength coverage was approximately $4500-7700 \AA$ with a spectral resolution of $6 \AA$. Comparison $\mathrm{Ne}-\mathrm{Hg}-\mathrm{Xe}$ lamps were taken every five positions to track instrument flexure. To simplify the observations, long-slits were generally not orientated along the parallactic angle. However, all observations were obtained at airmass $<1.4$ to limit the amount of light lost to atmospheric differential refraction.

For the remnant's NE and SW jets, along with some outer ejecta, a variety of instruments on the MDM $2.4 \mathrm{~m}$ were used. Some data were obtained using the Mark III Spectrograph (MKIII) employing a $11^{\prime \prime} 7 \times 4.5^{\prime}$ slit and using a SITe $1024 \times 1024$ CCD detector ('Templeton'). A 300 lines $\mathrm{mm}^{-1} 5400 \AA$ blaze grating yielded spectra spanning 4500-7400 $\AA$ with $10 \AA$ resolution. Other data were obtained using the Boller and Chivens CCD Spectrograph $(\mathrm{CCDS})$ with a $2^{\prime \prime} \times 4.5^{\prime}$ slit and either the (i) blue-sensitive 150 line $\mathrm{mm}^{-1} 4700 \AA$ blaze or (ii) red sensitive 158 line $\mathrm{mm}^{-1} 7530 \AA$ blaze grating. Both of these setups had approximately $12 \AA$ resolution. Comparison 


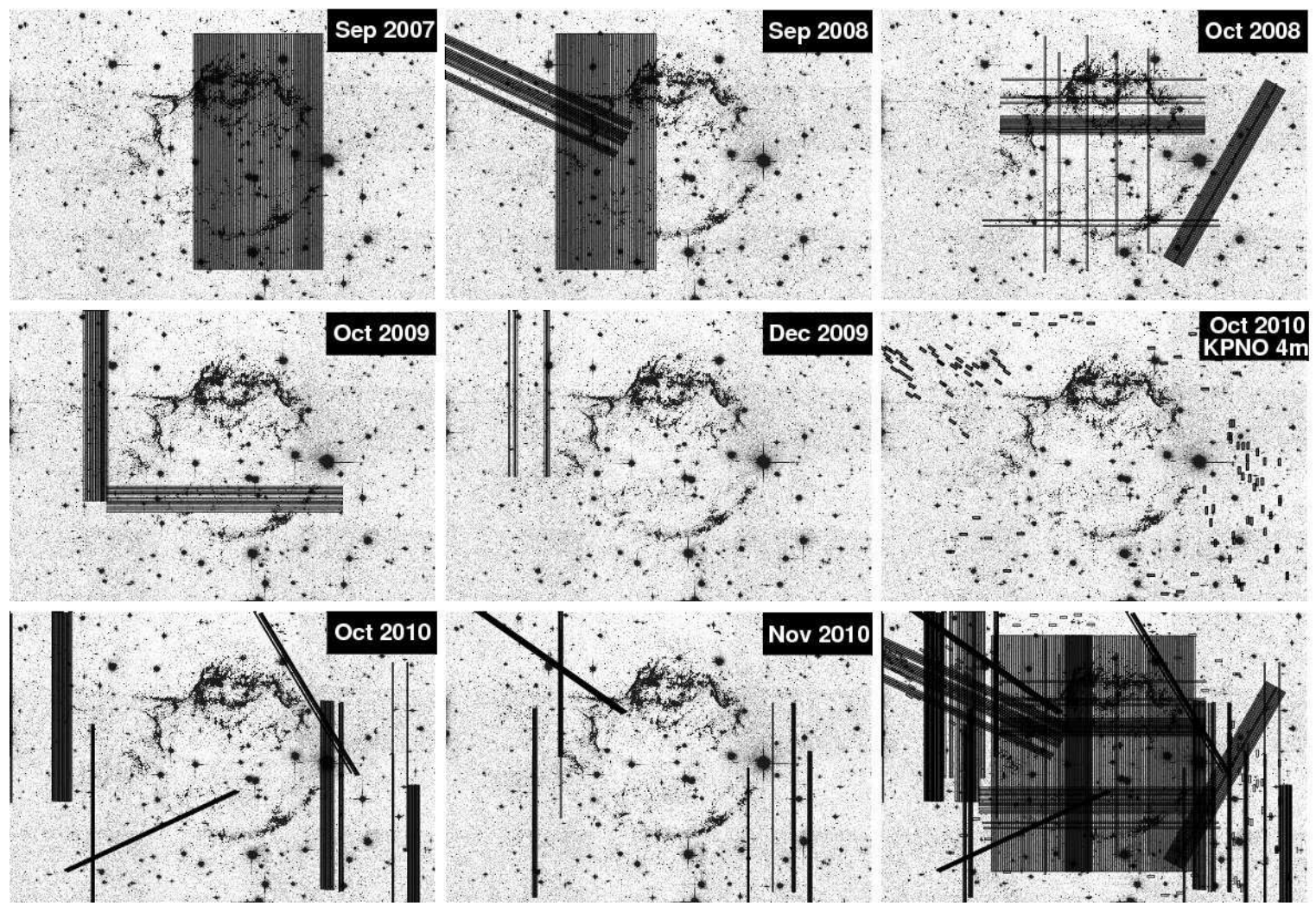

Figure 1. Finding charts of all long-slit positions. Background image is a mosaic created from 2004 HST/ACS observations sensitive to oxygen and sulfur emissions (Proposal 10286; PI: R. Fesen; see Fesen et al.|2006a). Bottom right is montage of all locations. Refer to Table 1 for telescope and instrument details.

Table 1

Summary of Observations

\begin{tabular}{llll}
\hline \hline \multicolumn{1}{c}{ Date } & \multicolumn{1}{c}{ Telescope } & Instrument & \multicolumn{1}{c}{ Region (No. of Positions) } \\
\hline 2007 Sep 01-08 & MDM 2.4 m & Modspec & Main Shell (58) \\
2008 Sep 18-25 & MDM 2.4 m & Modspec & Main Shell (45); NE Jet (18) \\
2008 Oct 27-30 & MDM 2.4 m & MKIII & Main Shell (19); SW Jet (10) \\
2009 Oct 19-22 & MDM 2.4 m & CCDS & Main Shell (9); NE Jet (16) \\
2009 Dec 11-14 & MDM 2.4 m & CCDS & NE Jet (6) \\
2010 Oct 1-5 & KPNO 4 m & MARS & NE/SW Jets + Outer Knots (81) \\
2010 Oct 8-13 & MDM 2.4 m & MKIII & NE/SW Jets + Outer Knots (38) \\
2010 Oct 28-Nov 1 & MDM 2.4 m & MKIII & NE/SW Jets + Outer Knots (26)
\end{tabular}

lamps and flats were taken to track telescope flexure and aid in the subtraction of fringing. These long-slit spectra were taken using longer exposures of $1000-2000$ s. Slits were oriented to maximize the number of ejecta knots detected at each slit position.

Standard stars from Stone (1977) and Massey \& Gronwall (1990) were observed each night. Comparison between evenings of each run generally showed good agreement, and for the main shell observing runs all standards were combined into one sensitivity function from which data were calibrated for relative flux. Some nights, particularly those for the outer ejecta knots, showed evidence of variable conditions and were flux calibrated independently on a night-by-night basis.

Multi-slit spectra of both NE and SW jet knots and other outlying ejecta were also obtained October 2010 with the Mayall 4m. The Multi-Aperture Red Spectrometer (MARS) was used in combination with the red- sensitive LBNL CCD detector and VPH 8050-450 grism. Spectra covered the wavelength region of $5500-10,8000$ $\AA$ with resolution of $\approx 8 \AA$. A total of 12 slit masks were made to cover 81 knots. We had 24 successful detections. Comparison He-Ne-Ar lamp images were taken for wavelength calibration, and standard star observations consistent with those made for the MDM runs were used for flux calibration.

\section{DATA REDUCTION}

Data were reduced homogeneously through a series of scripts written to automate IRAF/PyRAF 1 procedures. The 2D images of each position were first trimmed, bias-

1 IRAF is distributed by the National Optical Astronomy Observatories, which are operated by the Association of Universities for Research in Astronomy, Inc., under cooperative agreement with the National Science Foundation. PyRAF is a product of the Space Telescope Science Institute, which is operated by AURA for NASA. 


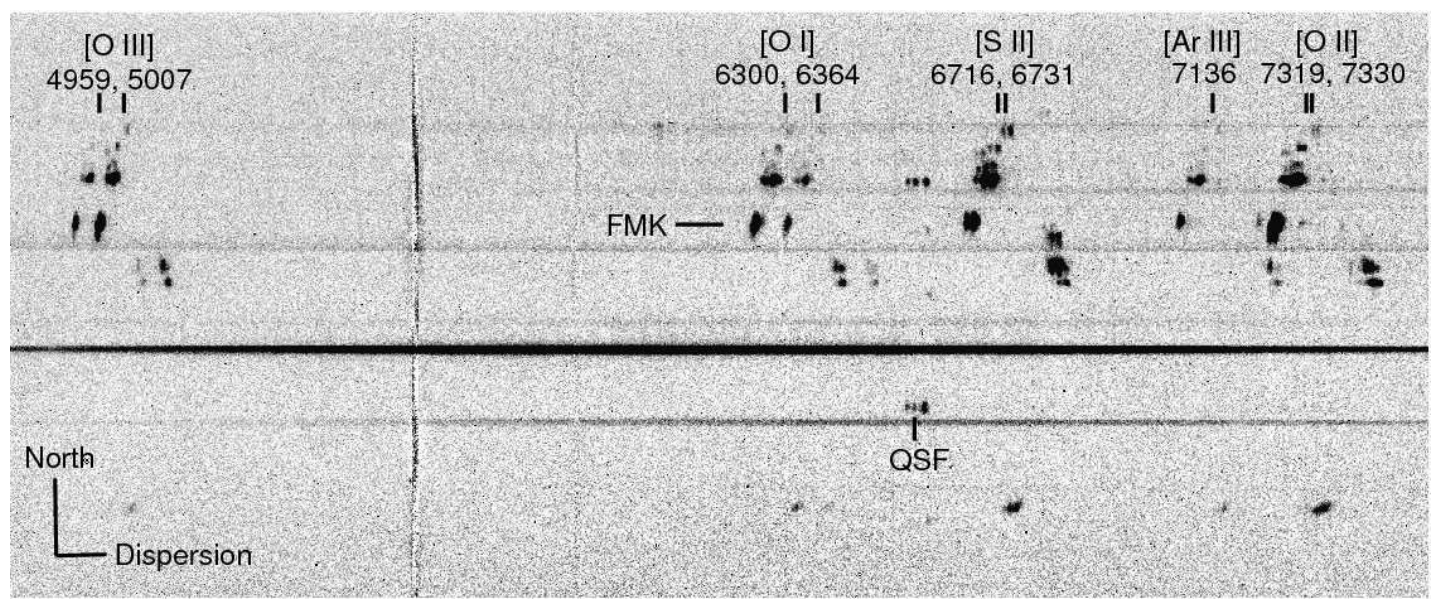

Figure 2. Example of a fully reduced and cleaned 2D spectrum of a slit position along the main shell from which 1D extractions were made. Main shell fast-moving knots (FMKs) and a CSM-related quasi-stationary material (QSF) are labeled. Wavelength positions for the brightest emission lines are marked.
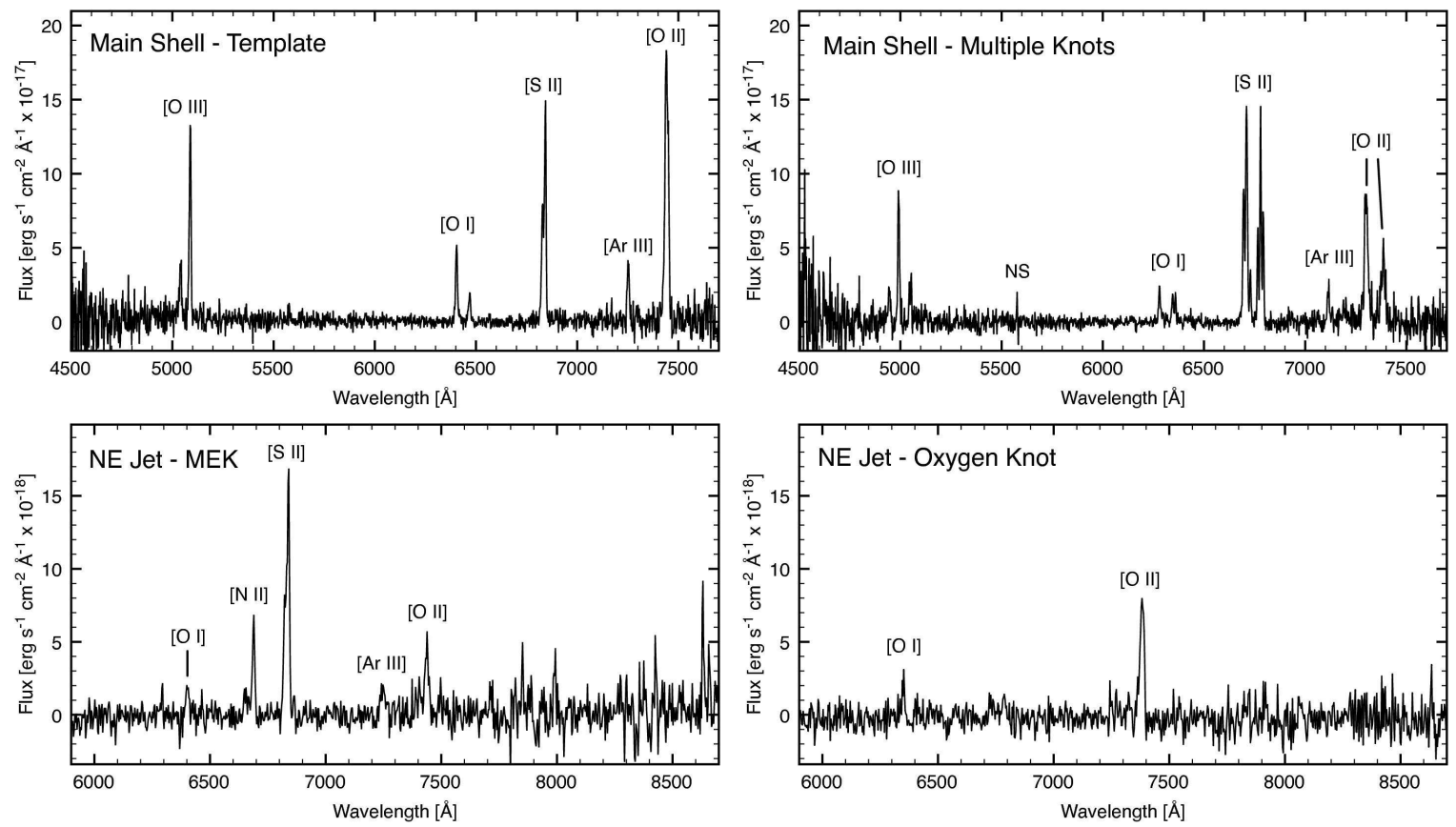

Figure 3. Examples of 1D spectra of ejecta knots of Cas A used in the Doppler reconstruction. The top left spectrum from the main shell is an example template used to identify knots and determine radial velocities. Top right shows a spectrum with multiple overlapping knots. Bottom left is a Mixed Ejecta Knot (MEK) from the NE Jet region. Bottom right is an oxygen-rich knot also from the NE Jet region.

subtracted, flattened, and co-added to remove cosmic rays. Images were then wavelength calibrated in the dispersion axis using the comparison lamp images and straightened in the spatial axis using tracings of stellar continua.

Excellent background subtraction of the images was achieved through the IRAF task background using a fifth-order chebyshev function fit along the spatial direction that was sampled from the median of 50 pixel bins. This provided sky emission clean 2D images from which 1D spectra could be extracted. An example of a reduced and cleaned 2D image is shown in Figure 2.

Each line of the 2D spectra was extracted and added in weighted triplet groupings. No effort was made to catalog the $\mathrm{H} \alpha,[\mathrm{N}$ II] $\lambda \lambda 6548,6583$, and He I emissions associated with circumstellar material
(CSM) often referred to as Quasi-Stationary Flocculi (QSF; van den Bergh \& Dodd 1970) frequently encountered (see Fig. 22). Investigation of this pre-SN mass loss material is left for future work.

From these 1D extractions, knot velocities were measured through cross-correlation of templates using the task xcsao. The template was based on actual data from a representative knot exhibiting the common lines of [O III] $\lambda \lambda 4959,5007,[\mathrm{O}$ I] $\lambda \lambda 6300,6364,[\mathrm{~S}$ II] $\lambda \lambda 6716$, 6731, [Ar III] $\lambda 7136$, and [O II] $\lambda \lambda 7319,7330$. We show example spectra of four types of ejecta knots encountered in Figure 3. xcsao was run on all extracted 1D spectra for Doppler velocities running between -6500 to $+6500 \mathrm{~km} \mathrm{~s}^{-1}$ to find individual knots. This procedure was found to work well even in cases with multiple knots (Figure 3, top right). 

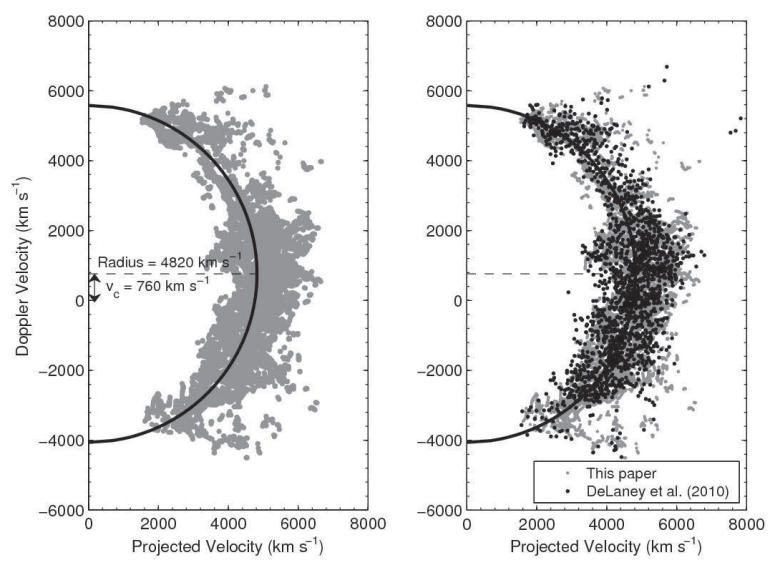

Figure 4. Measured main shell knot velocities. Projected radii from the COE have been converted to velocities using scale factor $S=0.022 \mathrm{~km} \mathrm{~s}^{-1} \operatorname{arcsec}^{-1}$. Left: Data from this paper. Best fit semi-circle is shown. Right: Data from DeLaney et al. (2010) for infrared Ar II emissions shown over top data from this paper.

Results of the preliminary selection were then manually inspected for false positives. The remnant's faint jet and other outlying ejecta knots required additional care owing to the range of emission lines expressed, such as mixed emission knots (Figure 3, bottom left), and oxygen-rich knots (Figure 3, bottom right). See Fesen \& Gunderson (1996) for additional details about the spectroscopic properties of these types of knots. In some cases velocities were determined manually using deconvolving techniques available in the task splot.

Radial velocities of individual knots were then assigned coordinates in right ascension (RA) and declination (DEC). A variety of cross-checks ensured consistent and accurate coordinates. Stars with well-measured coordinates encountered during the progression of longslit positions provided fudicial reference points. In some cases, bright stars were intentionally observed to provide stellar continua by which accurate positional offsets could be determined. Long-slit positions oriented east-west were checked against overlapping slits oriented north-south to further check positions and flux levels. A final check to all positions was aided through comparison with high-resolution Hubble Space Telescope (HST) images (Proposal 10286; PI: R. Fesen). Measured velocities are believed accurate to $\pm 40 \mathrm{~km} \mathrm{~s}^{-1}$ at the $68 \%$ confidence level. Positional uncertainties are estimated to be no more than $2^{\prime \prime}$; that is, of order the slit width.

\section{3D DOPPLER RECONSTRUCTION}

Cas A's relatively young age and nearby distance make it a prime candidate for Doppler velocity reconstruction. Previous studies show that its ejecta knots lie roughly on a spherical shell traveling radially outward from a unique center of expansion (COE; Reed et al. 1995; Lawrence et al. 1995; DeLaney et al. 2010). The COE is known to within one arcsecond precision and lies at the coordinates $\alpha(2000.0)=23{ }^{\mathrm{h}} 23^{\mathrm{m}} 27.77$ $\delta(2000.0)=+58^{\circ} 48^{\prime} 49^{\prime \prime} 4$ (Thorstensen et al. 2001). A lack of detectable deceleration in the proper motion of these knots over the last $50 \mathrm{yr}$ and velocity changes of less than $5 \%$ over 300 yr van den Bergh \& Dodd 1970; Kamper \& van den Bergh 1976; Thorstensen et al. 2001) allows one to assume ballistic trajectories from the
COE described by the relation

$$
v=r \times S,
$$

where $v$ is the radial velocity, $r$ is the angular distance from the explosion center, and $S$ is the scaling factor.

The value of $S$ can be found by fitting the measured Doppler velocities to a spherical expansion model. We follow procedures reviewed in DeLaney et al. (2010), which itself closely followed the work of Reed et al. (1995). The model is a semi-circle of the velocity distribution, which can be parameterized in terms of the center of the velocity distribution, $v_{c}$, the minimum velocity at which the semi-circle crosses the velocity axis, $v_{m}$, and $S$, which relates the velocity axis to the spatial axis as:

$$
\left(r_{p} / S\right)^{2}+\left(v_{D}-v_{c}\right)^{2}=\left(v_{c}-v_{m}\right)^{2},
$$

where $r_{p}$ is the observed projected radius and $v_{D}$ is the observed Doppler velocity.

In Figure 4, the results of our least-squares fit to the data are shown. The calculated Doppler velocities are $v_{c}=760 \pm 100 \mathrm{~km} \mathrm{~s}^{-1}, v_{m}=-4060 \pm 200 \mathrm{~km} \mathrm{~s}^{-1}$, and the scaling factor is $S=0^{\prime \prime} \cdot 022 \pm 0^{\prime \prime} .003$ per $\mathrm{km} \mathrm{s}^{-1}$. The results are consistent with those determined by Reed et al. (1995) using optical data, as well as with DeLaney et al. (2010) using Spitzer IR data of [Ar II] emission. Knot positions in RA and DEC were scaled to velocities using Equation 1. We used the COE and the calculated Doppler velocity $v_{c}$ to define a three-dimensional center of expansion in velocity space from which all reported vector trajectories originate.

\section{RESULTS}

The results from our survey encompassing 13,769 individual data points are presented in Figure 5. Incorporated into the data set are an additional 73 outer ejecta knots with measured radial velocities reported in Fesen (2001). The resulting Doppler maps have the highest spectral and spatial resolutions of any previous survey of Cas A, and represent the most complete catalog of its optically emitting ejecta material to date.

Measured knot radial velocities are represented in Figure 5 with a color-coded gradient. A surface reconstruction has been performed using a ball-pivoting algorithm to interpolate and smooth the original point cloud. The Doppler map is plotted against a background image of Cas A created from the HST ACS/WFC images (GO 10286; PI Fesen) obtained in filters F625W (colored blue), F775W (green), and F850LP (red) as a visual aid to relate the 3D kinematic structure to Cas A's appearance on the plane of the sky. Three viewing orientations are shown: a face-on view, a rear view, and an angled view from above shown with the background HST image offset. An animation has also been created showing the individual data points and the surface reconstruction, rotated along the north-south and east-west axes, with and without the outer high-velocity ejecta knots (Movie 1).

Below we discuss some specific properties of Cas A highlighting our high-resolution 3D reconstructions of the remnant's kinematic structure.

\subsection{Kinematic Properties of the Main Shell}




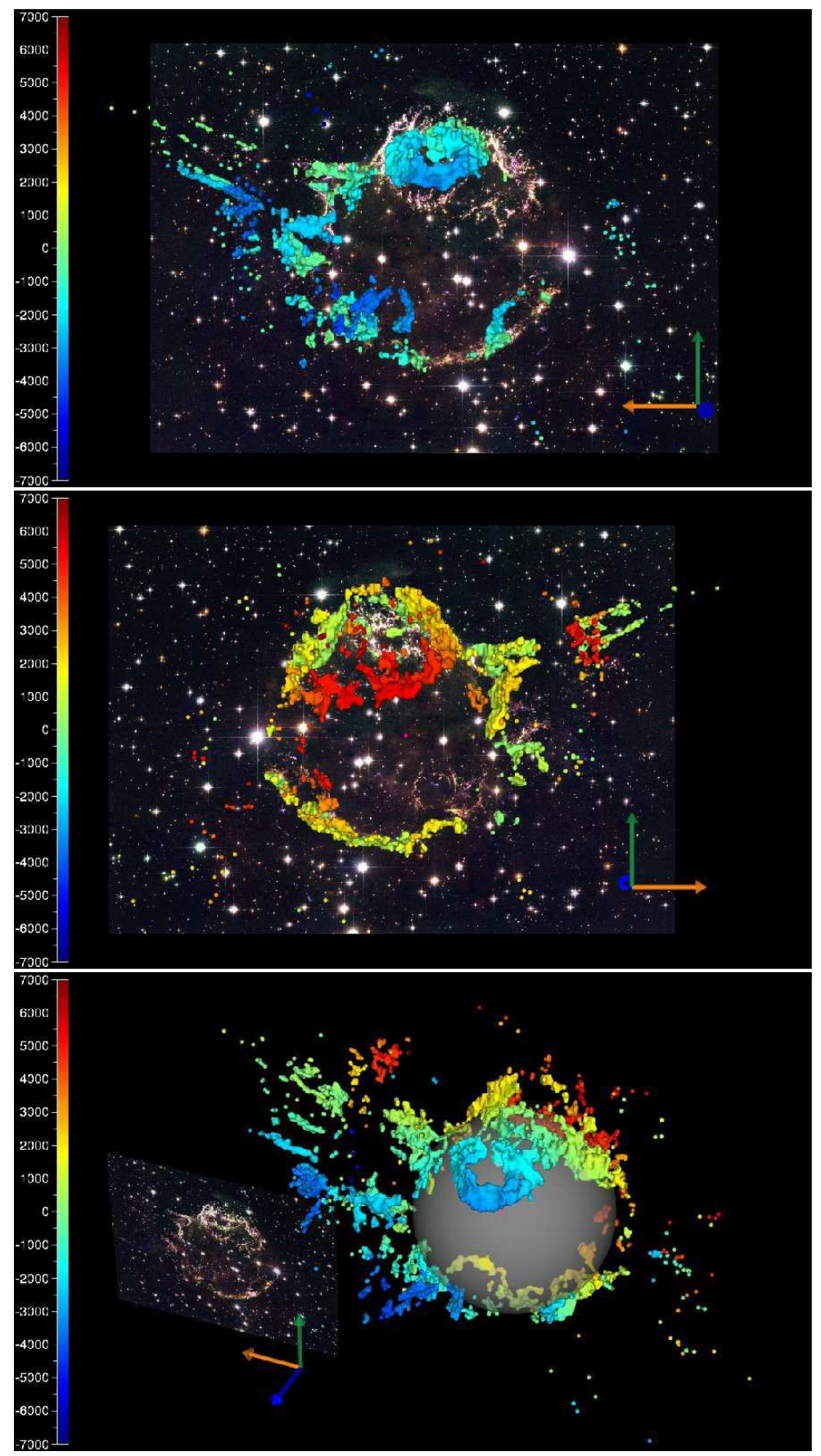

Figure 5. 3D Doppler reconstruction of Cas A's optically emitting ejecta. The main shell and all sampled high velocity outer material is represented. Top panel shows the reconstruction with respect to the plane of the sky as observed from Earth with north up and east to the left. The velocity gradient is color-coded and only blueshifted material is visible from this perspective. Middle panel shows the same perspective rotated $180^{\circ}$ with respect to the north-south axis. In this representation, the vantage point is from behind Cas A with only redshifted material shown. Bottom panel is an angled perspective showing the full range of velocities. The plane of the sky is shown offset for reference. A translucent sphere is a visual aid to help distinguish between front and back material. Refer to Movie 1 for an animation of these data.

The large-scale distribution of Cas A's main shell of ejecta is shown in Figure [6] where knots having an expansion velocity outside a $6000 \mathrm{~km} \mathrm{~s}^{-1}$ sphere have been excluded. Consistent with the recent $3 \mathrm{D}$ models presented in DeLaney et al. (2010) based on infrared data, we find the main shell to be dominated by morphological structures in the form of partial or complete rings. The main difference of our 3D reconstructions shown here to those of DeLaney et al. (2010) is increased spatial resolu- tion (by a factor of $\approx 4$ ) and more precise radial velocity data (by a factor of $\approx 5$ ).

We find at least six well-defined ring-like structures on the remnant's main shell with diameters between approximately $30^{\prime \prime}(0.5 \mathrm{pc})$ and $2^{\prime}(2 \mathrm{pc})$. In Figure 7 a Mercator projection of the main shell knots is shown to illustrate the relative scale and distribution of the rings. These rings dominate the large-scale structure of the main shell and are present on the front, rear, and 

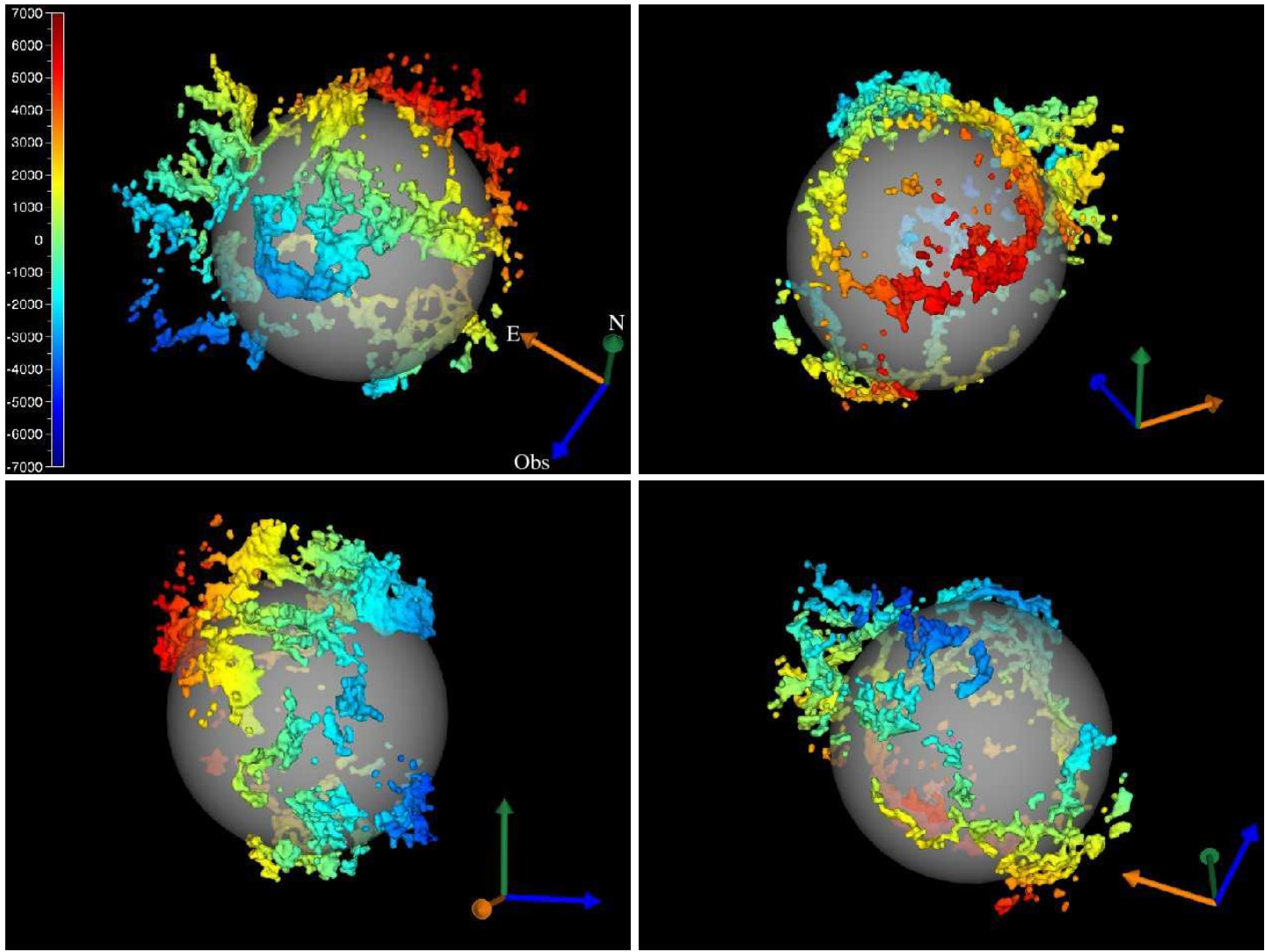

Figure 6. Various perspectives of the Cas A reconstruction illustrating the ubiquity of large ejecta rings. Only ejecta knots in the main shell are shown. Top left panel shows some of the more prominent ejecta structures. Noticeable rings of ejecta include the blueshifted ring in the north, and the much larger neighboring redshifted ring. Also seen is the extent to which the rings are extended radially and hence more crown-like than simple flat rings. The top right panel shows a better perspective of the large northern redshifted ring. Bottom left panel shows a side profile viewing towards the base of the NE jet, while the bottom right panel shows Cas A from below where a southwestern ring can be seen as well as a number of partial or broken ring structures. Refer to Movie 1 for an animation of these data.

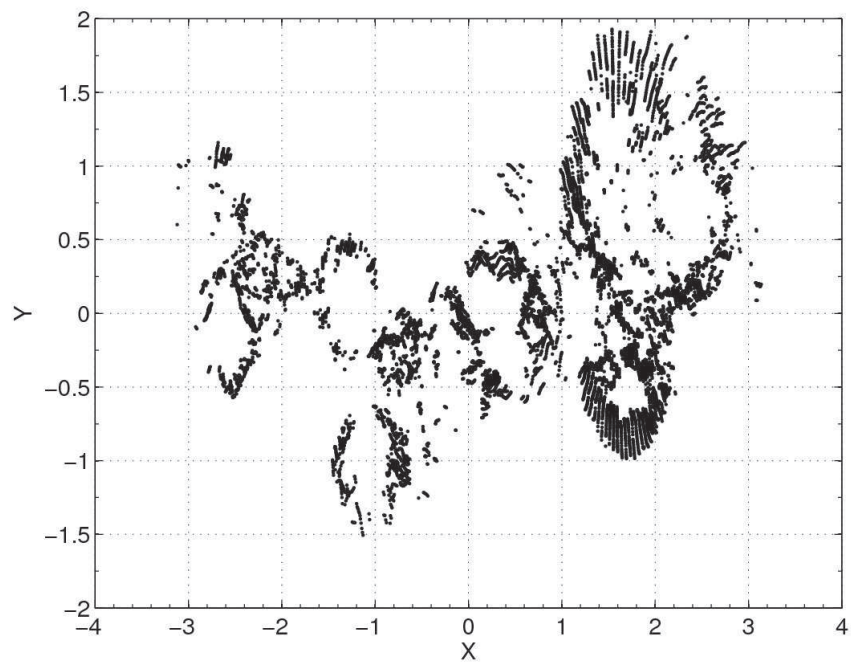

Figure 7. The main shell of Cas A's optically-emitting ejecta as represented in a Mercator projection. The linear scale is equal in all directions around any point and conformal, but the cylindrical map projection distorts the size and shape of large objects, especially towards the poles.

both east and west side hemispheres.

The largest ejecta ring is located along the northern limb and is nearly entirely redshifted (Figure 6] top right). Connected to it by a small bridge of ejecta knots is a much smaller but thicker ring in the north that is entirely blueshifted (Figure [6, top left). These two rings contain nearly all of the optical and infrared emission along the remnant's northern limb. A separate but also very large, outwardly extending broken ring is located near the base of the NE jet (Figure 6, bottom left). Another ring of comparable size and radial extension lies immediately below it.

Five of the six most obvious ejecta rings are actually short cylinders giving them a crown-like appearance. The bases of these crowns are sometimes gently curved upward as seen in the smaller northern blueshifted ring (Figure 6, top left), and the large northern ring (Figure6. top left and bottom left). The height in velocity space of these crowns that extend radially away from the COE is up to $1000 \mathrm{~km} \mathrm{~s}^{-1}$. The radial extent of the broken ring located near the base of the NE jet is largest (Figure 6. top right).

Besides large-scale ejecta rings and crowned cylinders, the data show areas with a frothy ejecta substructure down to sizes of $10^{\prime \prime}\left(\approx 5 \times 10^{17} \mathrm{~cm}\right)$. These smallscale features are often observed at the boundaries of larger rings in tightly arranged groupings. They can be distinguished in the Mercator projection in Figure 7 as the smallest rings and ellipses. Similar small-scale ringlike structures can be seen in HST images (Fig. 5 in Fesen et al. 2001).

The bulk of the remnant's optically bright main shell ejecta lies fairly close to the plane of the sky and can be contained within a torus or thick disk structure (see Figure 6] bottom left). With respect to a unit vector 

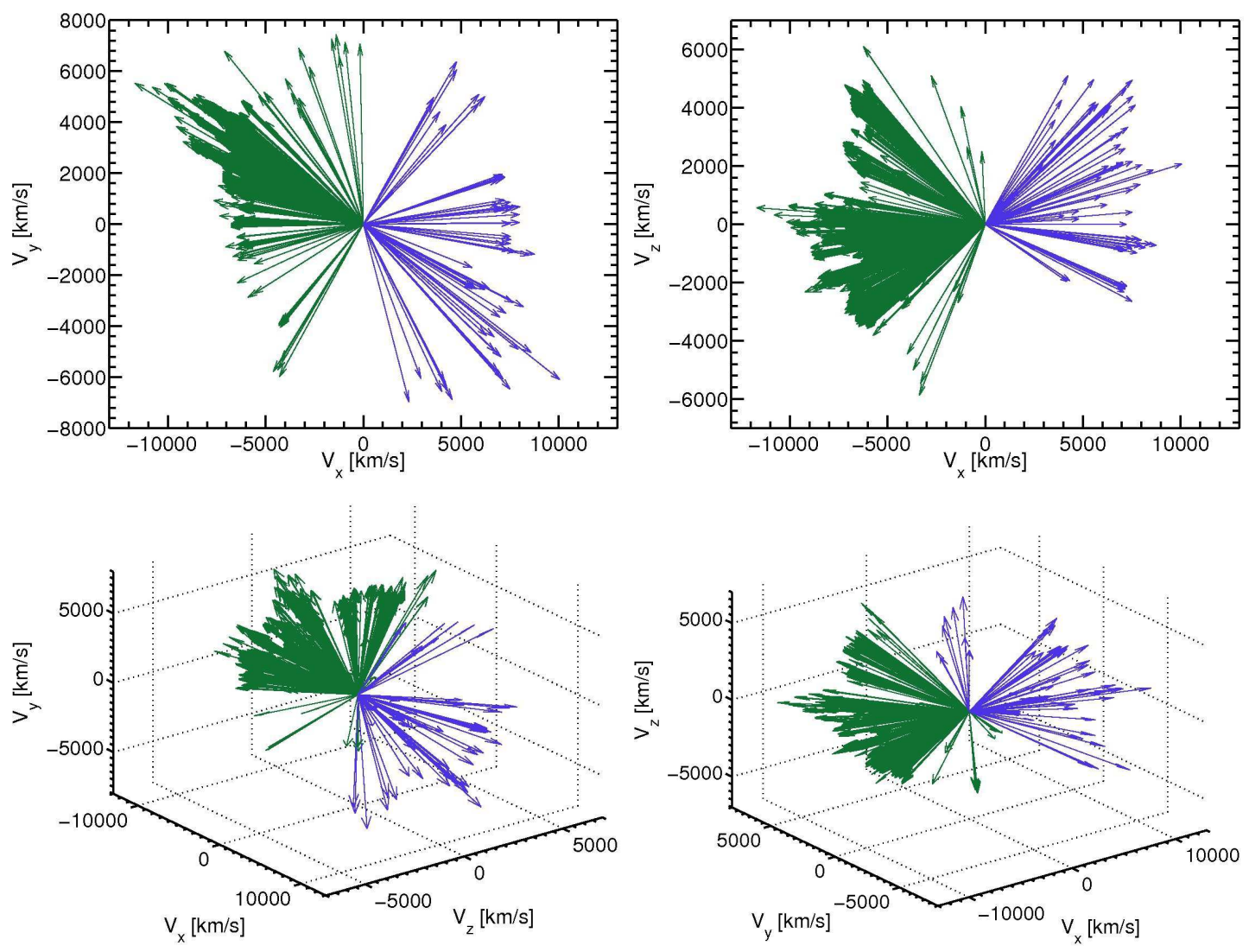

Figure 8. Vector plots of all sampled outer ejecta knots. $V_{x}$ is east-west, $V_{y}$ is north-south, and $V_{z}$ is radial velocity. The top left panel shows the perspective as observed from Earth, with north up and east to the left. All knots to the east are colored green, and all knots to the west are colored blue. The top right panel shows what the jets look like as viewed along the north-south axis looking toward south. Two angled perspectives are shown in the bottom left and right panels.

normal to the plane of the sky directed from the remnant to the observer, the vector normal to the torus' equatorial plane is tilted approximately $30^{\circ}$ to the west and $30^{\circ}$ to the north, in agreement to the findings of DeLaney et al. (2010). We note, however, that conspicuous deviations from a simple disk structure are observed. For example, the compact, entirely blueshifted northern ring extends beyond the torus defined by the bulk of the main shell. We discuss this and other deviations from a thick disk structure more in Section 6.2.

\subsection{The NE and SW High-Velocity Streams of Ejecta}

Vector plots of outer high velocity ejecta are shown in Figure 8 where ejecta knots are represented by green (east) and blue (west) vectors from the remnant's COE. An animation has been made that shows these data rotated completely about the north-south axis (Movie 2). Considerably more knots are detected in the NE than in the SW. This imbalance is may be attributable in part to higher levels of extinction in the SW compared to the NE. Fortunately, detections in the SW jet features are numerous enough to draw some conclusions about the relative properties of both high-velocity ejecta features.

Previous studies of the remnant's jets lacked the kinematic resolution and depth required to ascertain whether they form a true bipolar structure. Our data indicate that this is indeed the case. The NE and SW jet regions show a broad scattering of outlying, high-velocity knots that appear to be directed in nearly opposite directions and is suggestive of opposing flows of SN debris. Al- though the NE material exhibits a slightly larger range of radial velocities compared to the SW, we find no clear kinematic distinction between the two distributions in terms of opening half-angle and maximum expansion velocity.

The top left panel of Figure 8 shows the normal observer perspective of north up and east to the left. The distribution seen is much like the 'bowtie' plot of Fesen et al. (2006b) (their Fig. 4) created from measured proper motions of 1825 knots seen on $H S T$ images taken over a 9 month period. A straight line can be drawn that runs through the approximate center of both distributions, and this is maintained as the perspective is changed in the various panels of Figure 8.

These data reveal the brighter and richer NE jet's kinematic structure in much greater detail than previous studies. We find that instead of being a few thin streams of ejecta, the NE region actually encompasses a broad range of knot radial velocities including large redshifted velocities up to $+5000 \mathrm{~km} \mathrm{~s}^{-1}$. Prior spectroscopic data of the NE jet detected only a a handful of knots at these velocities (Fesen \& Gunderson 1996; Fesen 2001). Consequently, although the fastest knots lie close to the plane of the sky and thus exhibit relatively modest radial velocities, the NE jet's true structure is actually a rather broad fan of ejecta knots with an opening half-angle between $35-40^{\circ}$.

In Figure 9, four perspectives of the outer ejecta vectors with respect to the $\mathrm{COE}$ are shown along with the main shell reconstruction. The top two panels are in 

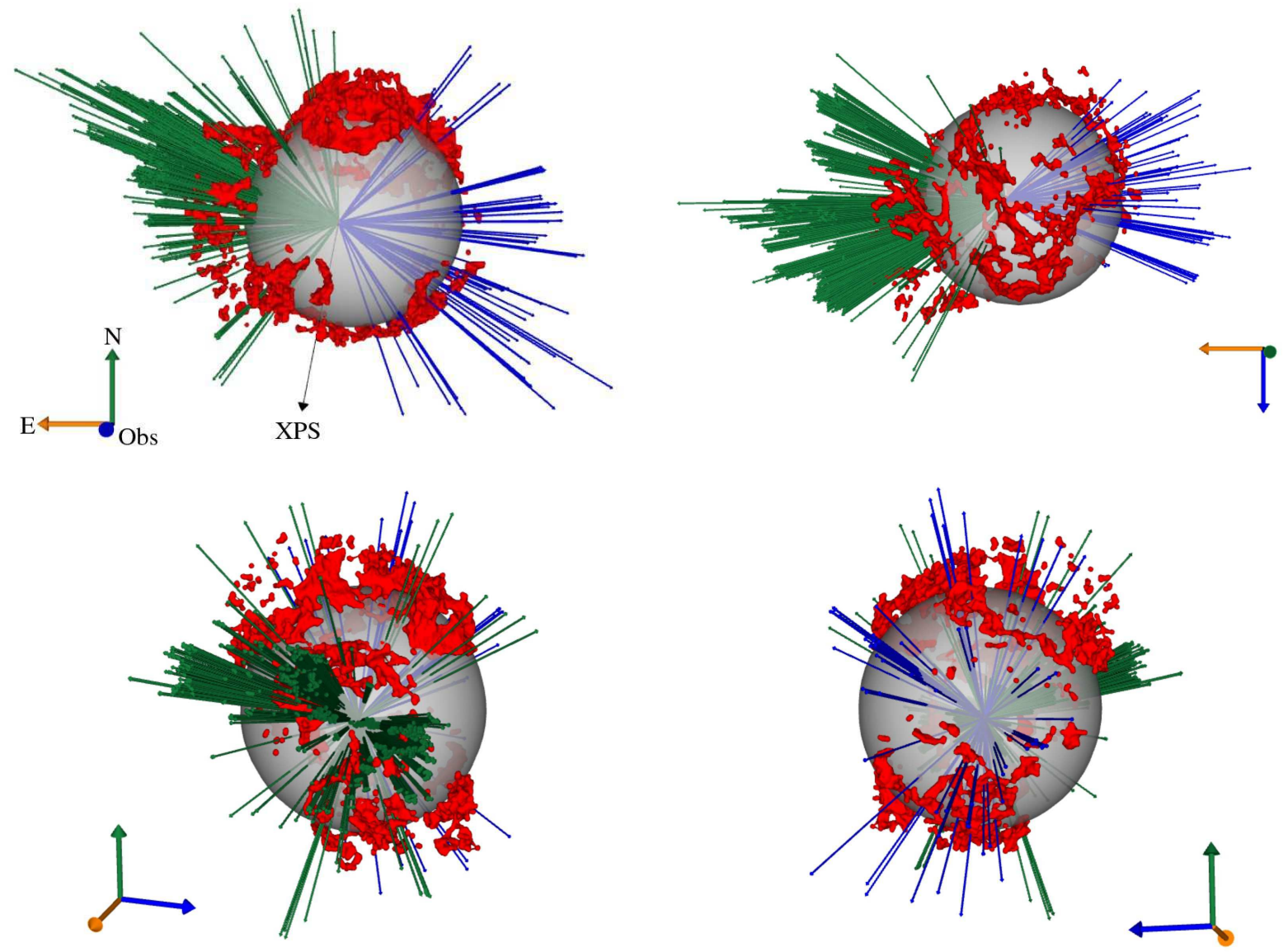

Figure 9. Vector plots of all NE and SW ejecta knots (colored green and blue, respectively) shown together with the main shell ejecta (red). Four angled perspectives are shown with the upper left being as seen in the sky. A black arrow shows the inferred motion of the XPS with respect to the center of expansion (Fesen et al. 2006b). Refer to Movie 2 for an animation of these data.

the same orientation as the top two panels of Figure 8 and use the same color scheme to distinguish the east and west material. The possible connection between the main shell geometry and distribution of outer ejecta is discussed in Section 6.3. Also shown in Figure 9, is the inferred motion of the X-ray point source (XPS) believed to be a neutron star with a carbon atmosphere (Ho \& Heinke 2009). The direction the XPS determined originally in Fesen et al. (2006b) is towards an obvious hole in the distribution of outer ejecta and is briefly discussed in Section 6.4.

\section{DISCUSSION}

Although optically-emitting debris may constitute only a small fraction of the total ejected mass in the Cas A remnant, our observations offer superior spatial and kinematic resolution compared to previous surveys obtained via Chandra X-ray and Spitzer infrared observations. Moreover, Cas A's fastest moving material is best probed through optical studies. For example, optical emission from the NE and SW jets can be traced some $90^{\prime \prime}$ farther out than in X-rays or infrared, and only a handful of outer optical ejecta knots around the remainder of the remnant are detected in even the deepest X-ray images of Cas A (see Fesen et al. 2006b).

It is now abundantly clear from the results of our survey and previous studies that the distribution of Cas A's ejecta is far from being random. At least half a dozen large and coherent ejecta ring-like structures are observed and their size and arrangement may be informing us about important properties of the explosion dynamics and subsequent evolution of the expanding debris. However, interpretation of these structures is complicated in that one must disentangle the observed remnant properties that may originate in the explosion from later influences related to possible post-explosion radioactive heating, ejecta interaction with the surrounding CSM and interstellar medium (ISM), and effects of the reverse shock on the ejecta.

An important additional caveat is that Cas A continues to evolve and has changed considerably in its optical appearance over the last 50 years that it has been monitored (Kamper \& van den Bergh 1976; van den Bergh \& Kamper 1985). On-going propagation of the remnant's reverse shock continues to excite new ejecta, revealing more of its entire distribution. Hence, any conclusions made in the present must acknowledge this evolution and recognize that we are probably not seeing the entire remnant but only those parts which have recently undergone shock heating.

With these limitations in mind, below we attempt to describe and understand the nature of the observed properties of Cas A's main shell and outlying high-velocity ejecta. 

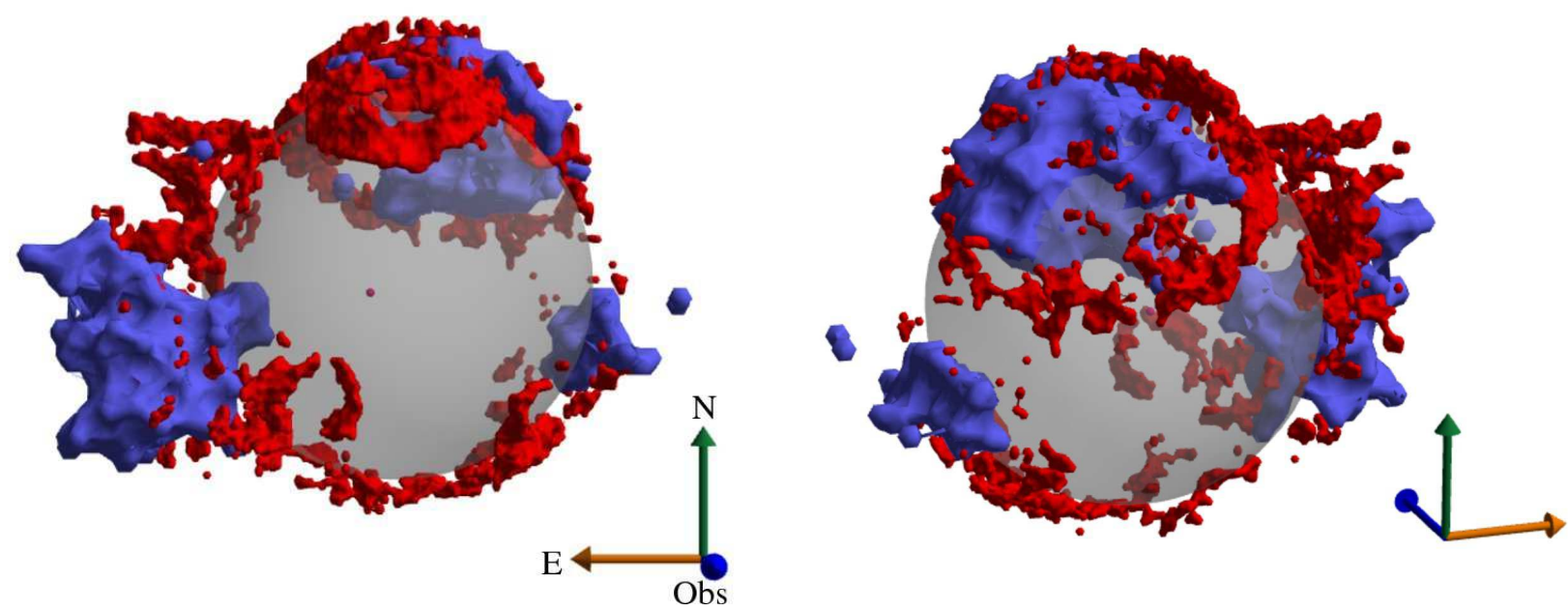

Figure 10. Location of iron-rich X-ray emitting ejecta (blue) with respect to the main shell sulfur- and oxygen-rich optically-emitting ejecta (red). The X-ray data shown is from DeLaney et al. (2010). Refer to Movie 3 for an animation of these data.

\subsection{Ejecta Rings $={ }^{56} \mathrm{Ni}$ Bubbles?}

Reed et al. (1995) and Lawrence et al. (1995) were the first to establish the existence of conspicuous rings of reverse shocked ejecta in Cas A. These rings, clearly visible in the recent infrared survey of DeLaney et al. (2010), are large (diameter $\sim 1 \mathrm{pc} \sim$ radius of Cas A) and are most certainly structures that are related to the true structure of the remnant's debris field and independent of any strong influences of the remnant's CSM/ISM environment. The optical data presented here does not alter this overall picture, but provides an improved, more detailed examination of Cas A's expansion properties.

In Figure 10, we show the optically-emitting main shell ejecta along with the location of X-ray emitting ironrich material measured from Chandra data presented in DeLaney et al. (2010). An animation showing these data sets rotated about the north-south and east-west axes has been provided (Movie 3). Each of the three largest concentrations of Fe-rich ejecta (i.e., along the west, north, and southeast limbs) lie within and bounded by ring structures, strongly suggesting a causal relationship.

DeLaney et al. (2010) noted the coincidence of large ejecta rings with the three regions of $\mathrm{Fe}-\mathrm{K} \mathrm{X}$-ray emission and argued that these and other less prominent features are regions where the ejecta have emerged from the explosion as 'pistons' of faster than average ejecta. In this view, the remnant's main shell rings represent the intersection points of these pistons with the reverse shock, similar to the bow-shock structures described by Braun et al. (1987).

An alternative explanation first suggested by Blondin et al. (2001) that we favor is that the observed ejecta rings represent cross-sections of large cavities in the expanding ejecta created in part by a post-explosion input of energy from plumes of radioactive ${ }^{56} \mathrm{Ni}$-rich ejecta. Li et al. (1993) have described how this input of energy might account for the highvolume filling factor of Fe in SN 1987A despite its small mass, and Basko (1994) and Blondin et al. (2001) have investigated hydrodynamic simulations based on this model. One possible consequence of this scenario is the compression of surrounding non-radioactive material by the rising and expanding bubbles of radioactive ${ }^{56} \mathrm{Ni}$-rich ejecta, ultimately giving way to a "Swiss cheese" ejecta structure. It is important to note that the main shell ejecta structures are observed as rings because we are biased by the reverse shock that only excites an thin shell of material. Thus, it is reasonable to suspect the observed ejecta rings to be cross sections of what are actually larger spherical geometries; i.e., bubbles.

The turbulent motions that would initiate this Ni bubble structure in Cas A are not unlike recent 3D simulations of the large-scale mixing that takes place in the shock-heated stellar layers ejected in the explosion of a $15.5 \mathrm{M}_{\odot}$ blue supergiant star presented in Hammer et al. (2010). As shown in their Figure 2, the progenitor's metal-rich core is partially turned over with nickel-dominated fingers overtaking oxygen-rich bullets. Although the evolution of these simulations is strongly dependent on the internal structure of the progenitor star (Ugliano et al. 2012), it is still tempting to draw an association between the Ni-rich outflows seen in the Hammer et al. (2010) models and the rings of Cas A.

However, there are difficulties with invoking a Ni bubble picture to explain how the X-ray emitting Fe ejecta are framed by rings of optical ejecta. Fe associated with the bubble effect should be characterized by diffuse morphologies and low ionization ages (Blondin et al. 2001; Hwang \& Laming 2003). The X-ray bright Fe we currently see, however, is actually at an advanced ionization age relative to the other elements and thus inconsistent with a $\mathrm{Ni}$ bubble origin.

It is possible that $\mathrm{Fe}$ associated with $\mathrm{Ni}$ bubbles remains undetected. The undetected Fe would likely be of small mass compared to what is currently observable since estimates of Cas A's chemical abundances are close to model predictions $\left(M_{\mathrm{Fe}} \sim 0.09-\right.$ $0.13 M_{\odot}$; Hwang \& Laming 2012), and be of low density (Eriksen et al. 2009). Presently, however, there is weak evidence of unshocked $\mathrm{Fe}$ ejecta in infrared data (DeLaney et al. 2010). Moreover, an exhaustive X-ray survey of Cas A by Hwang \& Laming (2012) led them to conclude that almost all of the Fe ejected by the supernova is now well outside the reverse shock and visible in $\mathrm{X}$-rays, with very little left in the center of the remnant. Indeed, any $\mathrm{Fe}$ associated with the bubble effect may 
be too faint and underionized to be readily identifiable (Hwang \& Laming 2003; Hwang \& Laming 2012).

Thus, while considerable evidence exists that argues against associating the X-ray bright Fe-rich material with $\mathrm{Ni}$ bubbles, the large-scale and coherent ejecta rings observed in Cas A are obviously not random filamentary ejecta structures, and their sizes, near spherical shapes, and ubiquity throughout the remnant are intriguingly suggestive of a Ni bubble origin. The strikingly tight spatial coincidence between some optical ejecta rings and the boundaries of Fe-rich ejecta seen in X-rays, perhaps best seen in the remnant's large northern ring of ejecta, lends support to this picture. Future efforts to locate additional material internal and/or external to Cas A's main shell may help clarify our understanding of the processes responsible for the ring-shaped morphology of the majority of Cas A's optically emitting ejecta.

Finally, we note that there is some evidence that expansion of radioactive Ni-rich ejecta may have also influenced the ejecta structure at smaller scales. A frothy ring-like substructure at scales of $10^{\prime \prime}$ (0.2 parsec) in highresolution HST images (Fesen et al. 2001) is observed around some of the large rings. These small features may be due to fragments of Ni-rich material pushing out surrounding material (e.g., blueshifted ring in Figure 6 , top left panel). However, this substructure should not be confused with possible influence from Rayleigh-Taylor and Kelvin-Helmhotz instabilities that can develop in clumpy ejecta due to their interaction with the reverse shock front. These processes may be the dominant ones behind the pronounced crown-like structure of the rings (cf. Basko 1994; Blondin et al. 2001).

\subsection{Main Shell Geometry and Velocity Asymmetry}

In addition to the large-scale ring structures of the main shell, there are two other observed properties of the main shell ejecta that may be related to the explosion dynamics. The first is the -4000 to $+6000 \mathrm{~km} \mathrm{~s}^{-1}$ radial velocity asymmetry. This asymmetry has long been recognized in even the earliest optical observations of the remnant made by Minkowski (1968), and later more fully appreciated by observations of Lawrence et al. (1995) and Reed et al. (1995). The second is the torus-like geometry observed for the bulk of the remnant's optical and infrared bright ejecta, discussed at length by Markert et al. (1983) and more recently by DeLaney et al. (2010).

Interpreting these properties is complicated given the problem of distinguishing whether the remnant's observed geometry is a faithful representation of the explosion dynamics or has been significantly influenced by an inhomogeneous local CSM and ISM. Reed et al. (1995) argued that environmental effects were significant in understanding the remnant's blue and redshift velocity asymmetry. They concluded that the density of the surrounding medium is greater on the blueshifted near side, which would inhibit forward expansion and result in an apparently redshifted COE. In support of this conclusion, they cite the observed asymmetrical distribution of radial velocities of the circumstellar QSF knots, a majority of which $(76 \%)$ are blueshifted.

However, DeLanev et al. (2010) and Isensee et al. (2010) note that there are major structural differences between the front and back interior surfaces of Cas A. These differences in brightness and structure could be due to different masses, densities, and energies in the two different directions suggesting the observed velocity asymmetry might be intrinsic to the explosion itself.

DeLaney et al. (2010) further argue that the torus-like distribution of bright main shell ejecta is a result of the explosion dynamics. They conclude that although interaction with the CSM affects the detailed appearance of the remnant, the bulk of the symmetries and asymmetries in Cas A are intrinsic to the explosion. In their framework, the remnant is the result of a flattened explosion where the highest velocity ejecta were expelled in a thick torus tilted not far off the plane of sky in a number of large-scale pistons.

In possible support of their picture, DeLaney et al. (2010) noted that Fesen (2001) found that relatively small angles off the sky plane (i.e., $<30 \mathrm{deg}$ ) seem to be the rule for the remnant's small, fastest-moving outlying ejecta knots. That is, although many hundreds of outlying ejecta knots can be seen around most of Cas A's periphery, none have been detected close to its center.

Our extensive survey also was unable to find any small, very high-velocity knots within the main shell and projected near the remnant's center. Rather than supporting a torus-like ejecta arrangement, however, this could indicate that a near tangent viewing angle may be important for a knot's optical visibility. Fesen (2001) originally proposed that a near tangent viewing factor might hold for both the remnant's outer knots and its main shell. If true, this would mean some portion of the remnant's facing and rear sides may remain undetected, and thus the currently observed torus-like configuration of bright emission not reflective of the remnant's true 3D structure.

The notion of a disk geometry for the Cas A remnant that happens to lie fairly near to the plane of the sky is also inconsistent with recent infrared observations that detected some of the remnant's interior, unshocked ejecta. Isensee et al. (2010) observed redshifted [Si III] and $[\mathrm{O}$ IV] line emissions from interior debris along a sight line close to remnant center exhibiting velocities approaching $5000 \mathrm{~km} \mathrm{~s}^{-1}$. These velocities are comparable to the -4000 to $+6000 \mathrm{~km} \mathrm{~s}^{-1}$ maximum radial velocities seen for main shell ejecta, as well as the inferred maximum transverse velocity of around $6300 \mathrm{~km}$ $\mathrm{s}^{-1}$ derived from proper motions $\left(\approx 0,0^{\prime \prime} 39 \mathrm{yr}^{-1}\right)$ of main shell ejecta knots assuming a remnant distance of 3.4 kpc (van den Bergh \& Kamper 1983; Thorstensen et al. 2001).

Further support of this viewpoint are some conspicuous departures from a thick-disk geometry in the distribution of main shell ejecta. Rear-facing ejecta seem to cut off abruptly along an angled plane, while the front-facing ejecta do not. In particular, the northern, blueshifted ring forms a conspicuous bulge from the apparent torus (see bottom left panel of Figure 6). There are aspects of the asymmetric distribution of the remnant's outermost ejecta that also indicate possible influence of an inhomogeneous environment. In Figure [11, the distribution of material as observed from two sides of Cas A looking toward the base of the NE and SW jets is shown. The material in the SW jet region largely follows the abrupt boundary cutoff, as does the main shell ejecta. Ejecta in the the NE jet region, however, do not follow this cut-off. Deep $\mathrm{H} \alpha$ images of Cas A show evidence for much more 


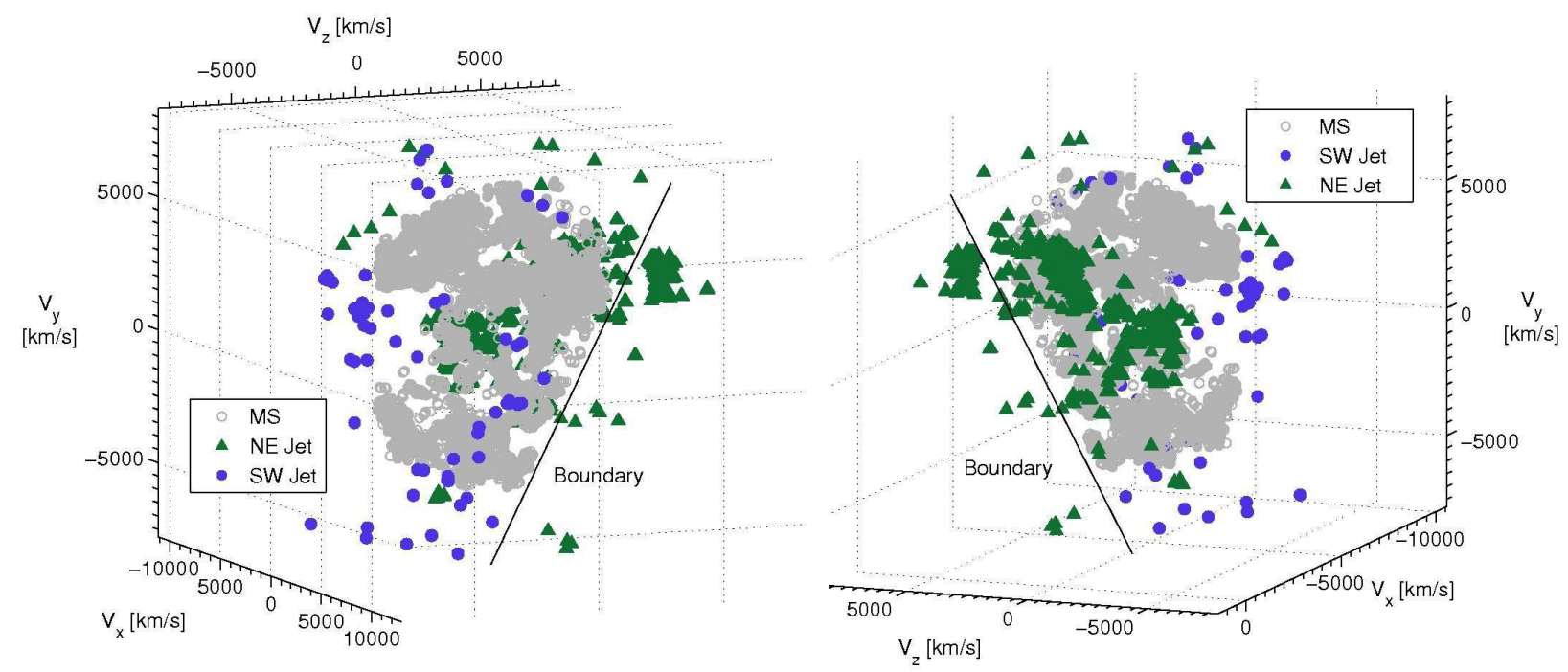

Figure 11. Side-looking perspectives of Cas A showing non-uniform distribution of material. The SW material (blue) and main shell ejecta (gray) cut off at a boundary defined by an angled plane. The NE material (green) does not cut off at the boundary, and this may due to interaction with nearby ISM/CSM H-rich material seen in $\mathrm{H} \alpha$.

H-rich CSM/ISM material in the eastern limb than in the western limb (Reynoso et al. 1997; Fesen 2001), which is consistent with the cut-off being due to environmental effects.

\subsection{Opposing High-Velocity Ejecta Outflows}

The nature of the anomalously high velocities of ejecta in the NE and SW regions has long been a puzzle. Minkowski (1968) suggested the NE jet might be the sole surviving part of an outer, high-velocity shell that has been subsequently decelerated in all other directions. Alternatively, because the distribution of emitting gas may not necessarily be the same as the actual distribution of gas in Cas A, the jets may simply be the most visible part of a larger population of the remnant's outer, high velocity knots (Fesen \& Gunderson 1996).

Along similar lines, asymmetric debris structures produced by circumstellar interaction has been theoretically modeled by Blondin et al. (1996) who show how a jetlike feature of SN ejecta can be generated in the progenitor's equatorial plane from pole/equator density gradients in the local CSM. Thus, these high-velocity regions may be secondary features caused by instability-powered flows from an equatorial torus, where the explosion axis is loosely defined by the X-ray iron-rich regions found in the southeast and northwest (Burrows et al. 2005; Wheeler et al. 2008; Rest et al. 2011).

However, the very prominent rupture-like features in the main shell near the base of the NE jet visible in X-ray, optical, IR, and radio images (cf. Hughes et al. 2000; van den Bergh \& Kamper 1985; Ennis et al. 2006; Anderson \& Rudnick 1995; DeLanev et al. 2010) are certainly suggestive of an explosive formation process. Although perhaps not indicative of a jet-induced explosion, there is nonetheless substantial evidence that the NE and SW jets are somehow associated with core-collapse explosion dynamics in some way.

For example, analyses of Chandra X-ray spectra indicate that the NE jet could not be formed through interaction with a cavity or lower density region in the CSM (Laming \& Hwang 2003; Laming et al. 2006). The remnant's ejecta would have expanded rapidly into a CSM cavity, and led to a density too low for electron-ion equilibration to match the observed values. Laming et al. (2006) estimate that a factor of two more explosion energy went into the NE jet direction compared to other regions.

The location of chemically distinct optically-emitting knots in both jets is also consistent with an unusual, high-velocity ejection of underlying mantle material. Knots exhibiting a mix of $\mathrm{H} \alpha$, [N II], [O II], [S II], and [Ar III] line emissions are only observed in the jet regions, suggesting an eruptive and turbulent mixing of the underlying $\mathrm{S}, \mathrm{O}$, and Ar rich material with photospheric H- and N-rich layers (Fesen \& Becker 1991; Fesen \& Gunderson 1996; Fesen 2001). Furthermore, optical knots in the NE and SW jets lying farthest out and possessing the highest ejection velocities show no detectable emission lines other than those of [S II], suggesting an origin from the S-Si-Ca-Ar rich layer deep inside the progenitor star (van den Bergh \& Kamper 1983; Fesen \& Gunderson 1996; Fesen 2001; Fesen et al. 2001).

As shown in Figure 8 , we find that the overall conical distributions of the $\mathrm{NE}$ and $\mathrm{SW}$ jet regions exhibit comparable maximum expansion velocities and are broadly anti-parallel in an orientation consistent with a bipolar jet-counterjet structure. However, the observed opening half-angles of these two flows $\left(\sim 40^{\circ}\right)$ is quite broad and is not what would be anticipated in a highly-collimated (opening half-angle $<10^{\circ}$ ) jetinduced explosion. Thus, the broadness and estimated low energies of the NE and SW jets make it unlikely that they are signatures of a jet-induced explosion scenario (e.g., Khokhlov et al. 1999; Wheeler et al. 2002; Akivama et al. 2003; Fryer \& Warren 2004; Laming et al. 2006).

There appears to be only a tenuous connection between the NE and SW jet regions and any of the main shell's ejecta rings. The centers of cones broadly defining the NE and SW ejecta streams as sampled in our survey only loosely point to centers of ejecta rings. The respective distributions are illustrated in Figure 9, where the outer knot vectors are plotted with the main shell surface 
reconstruction. Particularly suggestive is the NE jet region, where the bulk of high-velocity material appears to be emanating from a large main shell ring that has a pronounced radial extension. In this regard, the main shell rings and the jet-like streams of higher velocity debris could be dynamically related.

However, for both the NE and SW jets, many knots have trajectories that do not pass through any main shell rings. In light of the large number of faint, outermost high-velocity jet knots, it is possible that additional material at even higher positive and negative radial velocities may lie undetected, broadening the jets even more. Future work mapping the chemical abundances of jet knots to their kinematics might contribute to a better understanding of their nature and possible relationship to the main ejecta shell.

\subsection{Motion of X-ray Point Source}

An additional clue to the nature of the explosion dynamics may come from understanding the properties of the inferred motion of the central XPS. The location of the $\mathrm{XPS} \approx 7^{\prime \prime}$ to the southeast of the estimated COE (P.A. $\approx 170^{\circ}$ ) indicates a transverse velocity 'kick' of $\approx 350 \mathrm{~km} \mathrm{~s}^{-1}$ imparted to the compact object during the explosion (Fesen et al. 2006b).

Intriguingly, the projected line connecting the NE and SW jets lies nearly perpendicular to the inferred direction of the XPS (see Figure 8). Assuming the NE/SW axis to be the most significant in the original core-collapse, this runs counter to most jet-induced explosion models that predict that the neutron star will undergo a kick roughly aligned with the jet axis (Burrows \& Haves 1996; Fryer \& Warren 2004).

There is a noticeable absence of outlying ejecta along the axis of the XPS's inferred motion (Fesen 2001; Fesen et al. 2006b). With the possible exception of unlikely and extremely localized extinction conditions, deep imaging of Cas A surveying the entire remnant ensures that there is no observational bias that could explain the lack of detection of ejecta in these regions. Although possibly just a coincidence, it is interesting that the XPS should be moving with a trajectory in line with the only two significant gaps of the outer ejecta which are located nearly perpendicular to the NE-SW jet axis.

We note that Smith et al. (2009) showed that strong [Ne II] emission is observed symmetrically around the XPS, with an axis also close to the direction of the inferred kick. Although Smith et al. (2009) determined that there is not enough mass/energy in the Ne-crescents to affect the XPS motion, they may perhaps arise due to the same dynamical asymmetries that led to the XPS motion (DeLaney et al. 2010).

\subsection{Comparison to Other Young SNRs}

Recent 3D reconstructions of other young CCSN remnants have suggested that many of the kinematic properties observed in Cas A are not unique. Although only a handful of known SNRs are appropriate for this kind of analysis, those that have been studied have revealed evidence of the same large-scale ejecta rings, velocity asymmetries, and high-velocity bipolar asymmetries as observed in Cas A.

For example Vogt \& Dopita (2010) mapped the [O III] $\lambda 5007$ line emission dynamics of the young oxygen-rich supernova remnant 1E 0102.2-7219 in the Small Magellanic Cloud. They confirmed earlier Doppler measurements of Eriksen et al. (2001) that showed the presence of large ejecta rings like those observed in Cas A. Also like in Cas A, they found 1E 0102.2-7219 to have an overall velocity asymmetry in its bulk material, such that redshifted material contained fainter clumps but with higher velocities than the blueshifted material.

A similar [O III] $\lambda 5007$ survey of the young oxygenrich supernova remnant N132D in the Large Magellanic Cloud by Vogt \& Dopita (2011) also uncovered properties similar to Cas A. They found the majority of the ejecta to form a ring of $\sim 12 \mathrm{pc}$ in diameter inclined at an angle of $\sim 25^{\circ}$ to the line of sight, and evidence of a polar jet associated with a very fast oxygen-rich knot. The survey led Vogt \& Dopita (2011) to speculate that the overall observed shape of the SNR to have been strongly influenced by the pre-supernova mass loss from the progenitor star.

Work by Winkler et al. (2009) on the core-collapse SNR G292.0+1.8 shows that it too has properties like those seen in Cas A. Proper motion measurements of G292.0+1.8's fast filaments exhibit systematic motions outward from a point near the center of the radio/Xray shell that lies $46^{\prime \prime}$ northwest from the young pulsar PSR J1124-5916. The inferred motion of the pulsar has a transverse velocity of $440 \mathrm{~km} \mathrm{~s}^{-1}$, which is close to that observed in Cas A's XPS. Additionally, the fastest ejecta in G292.0+1.8 form a bipolar outflow along an axis oriented roughly north-south in the plane of the sky. The remnant appears to have undergone a complex evolution resulting from a possibly asymmetric SN explosion and interaction with non-uniform ambient ISM (see also Braun et al. 1986; Park et al. 2002), which is a scenario not too different from the one we envision for Cas A.

\subsection{Connections to Extragalactic SN Observations}

Finally, we consider how the kinematic properties of Cas A discussed here may be linked to phenomena observed in the late-time optical emissions of extragalactic supernovae. Milisavlievic et al. (2012) summed all main shell spectra presented in Section 2 into a single, integrated spectrum mimicking what the remnant would appear as as an unresolved extragalactic source. Similarities were seen between this integrated Cas A spectrum and several late-time optical spectra of decades-old extragalactic SNe.

Particularly well-matched with Cas A were SN 1979C, SN 1993J, SN 1980K, and the ultra-luminous supernova remnant in NGC 4449. Milisavljevic et al. (2012) found that both Cas A and decades old extragalactic SNe show pronounced blueshifted emission with conspicuous line substructure in [O I], [O II], [O III], [S II], and [Ar III]. Since the emission line substructure observed in the forbidden oxygen emission line profiles of Cas A are associated with the large-scale rings of ejecta, we suggest that identical features in the intermediate-aged SNe which have often been interpreted as ejecta 'clumps' or 'blobs' are, in fact, probable signs that large-scale rings of ejecta are common in SNe. The link is strongest between Cas A and SN 1993J, given that these were both Type IIb explosions and that they exhibit extremely similar forbidden oxygen profiles despite the three centuries of evolution that separate them. 


\section{CONCLUSIONS}

We have presented 3D kinematic reconstructions of the optical emission from the supernova remnant Cas A based on radial velocity measurements extracted from long-slit and multi-slit spectra. This data set is of high spatial and kinematic resolution and encompasses the NE and SW streams of high velocity ejecta jets that until now have never been surveyed to this depth.

The major results and conclusions of this study are as follows:

1) We confirm the findings of several previous kinematic studies of Cas A that show the bulk of the remnant's main shell ejecta to be arranged in several welldefined complete or broken ring-like structures. These ring structures have diameters that can be comparable to the radius of the remnant $(\sim 1 \mathrm{pc})$. Some rings show considerable radial extensions giving them a crown-like appearance, while other rings exhibit a frothy, ring-like sub-structure on scales of $\sim 0.2 \mathrm{pc}$.

Such large-scale ejecta rings may be a common phenomenon of young, core-collapse supernova remnants. Evidence for this comes in part from lumpy emission line profile substructure seen in both an integrated Cas A spectrum and several late-time optical spectra of extragalactic supernovae obtained years to decades after explosion. Because this line substructure can be directly mapped to the large-scale rings of our Cas A reconstruction, we suggest a similar origin may apply to unresolved extragalactic remnants.

2) The bulk of Cas A's optically bright ejecta populate a torus-like geometry that is tilted approximately $30^{\circ}$ with respect to the plane of the sky and exhibit a -4000 to $+6000 \mathrm{~km} \mathrm{~s}^{-1}$ radial velocity asymmetry. Unlike the conclusion reached by DeLaney et al. (2010), we suggest that this observed geometry and velocity asymmetry is not representative of the true overall kinematic properties of the original explosion. Instead, an observational bias caused by a near tangent viewing angle effect and interaction with an inhomogeneous CSM/ISM environment has likely contributed to some of Cas A's presently observed kinematic properties.

3) The size, shape, and ubiquity of large-scale, reverse shock heated ejecta rings in Cas A are consistent with a bubble-like interior structure. It is possible that some of these structures were generated by the input of energy from radioactive ${ }^{56} \mathrm{Ni}$ rich ejecta that produced low density bubbles surrounded by non-radioactive, intermediate mass element-rich ejecta. While the spatial coincidence between X-ray emitting Fe-rich material and some of the remnant's large ejecta rings is suggestive of such a origin, the high ionization age of the X-ray bright Fe suggests that this material is not directly associated with Ni bubbles.

4) Our deep optical survey shows the remnant's NE and SW jet features, which contain Cas A's highest velocity ejecta, to be unexpectedly broad streams of ejecta knots with comparable conical opening half-angles of approximately $40^{\circ}$. The jets appear to lie in an orientation consistent with an opposing and wide bipolar outflow. The broadness together with low energy estimates of the NE and SW jets argue against them being associated with a jet-induced explosion. However, several kinematic and chemical properties support the view that they were formed during core collapse and independent of effects from the local CSM or ISM. Faint ejecta in both jets at even higher expansion velocities may lie as yet undetected.

We end by noting that similar radial velocity data sets spanning X-ray, optical, and infrared wavelengths of other young SN remnants may provide additional insights and tests of various core-collapse SN models. A comparison of Cas A's properties with those of other young remnants, especially ones supplemented with chemical abundance analyses in addition to kinematics, may also help to further constrain details of the explosion mechanism, post-explosion dynamics, ejecta instabilities, and explosive nucleosynthesis. With the growing success obtaining multi-perspective optical spectra of light echoes associated with other historical Galactic supernovae e.g., SN 1572 (Krause et al. 2008), and those nearby in the LMC and SMC (Rest et al. 2005), the possibility of developing $3 \mathrm{D}$ reconstructions of other young CCSN supernova remnants with known SN subtypes may be possible in the future.

\section{ACKNOWLEDGMENTS}

We thank an anonymous referee for comments that improved this paper's content and presentation. D. Patnaude, R. Chevalier, J. Thorstensen and G. Wegner provided helpful discussions and comments. T. DeLaney kindly provided infrared and X-ray data in advance of publication. David Adalsteinsson provided considerable support with the DataTank software (http://www.visualdatatools.com), which was used to prepare some figures and the animations. Surface reconstruction was aided with the use of MeshLab (http://meshlab.sourceforge.net), a tool developed with the support of the 3D-CoForm project. This material is based upon work supported by the National Science Foundation under Grant No. AST-0908237.

\section{REFERENCES}

Akiyama, S., Wheeler, J. C., Meier, D. L., \& Lichtenstadt, I. 2003, ApJ, 584, 954

Anderson, M. C., \& Rudnick, L. 1995, ApJ, 441, 307

Basko, M. 1994, ApJ, 425, 264

Besel, M.-A., \& Krause, O. 2012, A\&A, 541, L3

Blondin, J. M., Borkowski, K. J., \& Reynolds, S. P. 2001, ApJ, 557, 782

Blondin, J. M., Lundqvist, P., \& Chevalier, R. A. 1996, ApJ, 472, 257

Blondin, J. M., Mezzacappa, A., \& DeMarino, C. 2003, ApJ, 584, 971

Braun, R., Goss, W. M., Caswell, J. L., \& Roger, R. S. 1986 A\&A, 162, 259

Braun, R., Gull, S. F., \& Perley, R. A. 1987, Nature, 327, 395

Burrows, A., Dessart, L., Ott, C. D., \& Livne, E. 2007,

Phys. Rep., 442, 23

Burrows, A., \& Hayes, J. 1996, Physical Review Letters, 76, 352

Burrows, A., Hayes, J., \& Fryxell, B. A. 1995, ApJ, 450, 830

Burrows, A., Livne, E., Dessart, L., Ott, C. D., \& Murphy, J. 2006, ApJ, 640, 878

Burrows, A., Walder, R., Ott, C. D., \& Livne, E. 2005, The Fate of the Most Massive Stars, 332, 350

Chevalier, R. A. 2005, ApJ, 619, 839

Chevalier, R. A., \& Kirshner, R. P. 1979, ApJ, 233, 154

Chevalier, R. A., \& Oishi, J. 2003, ApJ, 593, L23

Claeys, J. S. W., de Mink, S. E., Pols, O. R., Eldridge, J. J., \& Baes, M. 2011, A\&A, 528, A131

Delaney, T. A. 2004, PhD thesis, Univ. of Minnesota 
DeLaney, T., Rudnick, L., Stage, M. D., et al. 2010, ApJ, 725, 2038

Ennis, J. A., Rudnick, L., Reach, W. T., et al. 2006, ApJ, 652, 376

Eriksen, K. A., Arnett, D., McCarthy, D. W., \& Young, P. 2009, ApJ, 697, 29

Eriksen, K. A., Morse, J. A., Kirshner, R. P., \& Winkler, P. F. 2001, Young Supernova Remnants, 565, 193

Fesen, R. A. 2001, ApJS, 133, 161

Fesen, R. A., \& Becker, R. H. 1991, ApJ, 371, 621

Fesen, R. A., \& Gunderson, K. S. 1996, ApJ, 470, 967

Fesen, R. A., Morse, J. A., Chevalier, R. A., et al. 2001, AJ, 122, 2644

Fesen, R. A., Zastrow, J. A., Hammell, M. C., Shull, J. M., \& Silvia, D. W. 2011, ApJ, 736, 109

Fesen, R. A., Hammell, M. C., Morse, J., et al. 2006a, ApJ, 636, 859

Fesen, R. A., Hammell, M. C., Morse, J., et al. 2006b, ApJ, 645, 283

Fryer, C. L., \& Warren, M. S. 2004, ApJ, 601, 391

Hammer, N. J., Janka, H., \& Müller, E. 2010, ApJ, 714, 1371

Hanke, F., Marek, A., Müller, B., \& Janka, H.-T. 2012, ApJ, 755,

Hines, D. C., Rieke, G. H., Gordon, K. D., et al. 2004, ApJS, 154, 290

Ho, W. C. G., \& Heinke, C. O. 2009, Nature, 462, 71

Hughes, J. P., Rakowski, C. E., Burrows, D. N., \& Slane, P. O. 2000, ApJ, 528, L109

Hwang, U., \& Laming, J. M. 2012, ApJ, 746, 130

Hwang, U., \& Laming, J. M. 2003, ApJ, 597, 362

Hwang, U., Laming, J. M., Badenes, C., et al. 2004, ApJ, 615, L117

Isensee, K., Rudnick, L., DeLaney, T., et al. 2010, ApJ, 725, 2059

Janka, H.-T. 2012, Annual Review of Nuclear and Particle Science, 62,407

Janka, H.-T., Buras, R., \& Rampp, M. 2003, Nuclear Physics A, 718,269

Janka, H., Langanke, K., Marek, A., Martínez-Pinedo, G., \& Müller, B. 2007, Phys. Rep., 442, 38

Kamper, K., \& van den Bergh, S. 1976, ApJS, 32, 351

Keohane, J. W., Rudnick, L., \& Anderson, M. C. 1996, ApJ, 466, 309

Khokhlov, A. M., Höflich, P. A., Oran, E. S., et al. 1999, ApJ, 524, L107

Kifonidis, K., Plewa, T., Janka, H., \& Müller, E. 2000, ApJ, 531, L123

Kifonidis, K., Plewa, T., Janka, H., \& Müller, E. 2003, A\&A, 408, 621

Kjær, K., Leibundgut, B., Fransson, C., Jerkstrand, A., \& Spyromilio, J. 2010, A\&A, 517, A51

Kotake, K., Yamada, S., \& Sato, K. 2005, ApJ, 618, 474

Krause, O., Birkmann, S. M., Usuda, T., et al. 2008, Science, 320, 1195

Krause, O., Tanaka, M., Usuda, T., et al. 2008, Nature, 456, 617

Laming, J. M., \& Hwang, U. 2003, ApJ, 597, 347

Laming, J. M., Hwang, U., Radics, B., Lekli, G., \& Takács, E. 2006, ApJ, 644, 260

Lawrence, S. S., MacAlpine, G. M., Uomoto, A., et al. 1995 AJ, 109, 2635

Li, H., McCray, R., \& Sunyaev, R. A. 1993, ApJ, 419, 824

Maeda, K., Kawabata, K., Mazzali, P. A., et al. 2008 Science, 319, 1220

Marek, A., \& Janka, H.-T. 2009, ApJ, 694, 664

Markert, T. H., Clark, G. W., Winkler, P. F., \& Canizares, C. R. 1983, ApJ, 268, 134
Masada, Y., Takiwaki, T., Kotake, K., \& Sano, T. 2012, ApJ, 759,110

Massey, P., \& Gronwall, C. 1990, ApJ, 358, 344

Mazzali, P. A., Kawabata, K. S., Maeda, K., et al. 2005

Science, 308, 1284

Mazzali, P. A., Kawabata, K. S., Maeda, K., et al. 2007, ApJ, 670,592

Milisavljevic, D., Fesen, R. A., Chevalier, R. A., et al. 2012, ApJ, 751,25

Milisavljevic, D., Fesen, R. A., Gerardy, C. L., Kirshner, R. P., \& Challis, P. 2010, ApJ, 709, 1343

Minkowski, R. 1959, URSI Symp. 1: Paris Symposium on Radio Astronomy, 9, 315

Minkowski, R. 1968, Nonthermal Galactic Radio Sources, ed. Middlehurst, B. M. \& Aller, L. H. (the University of Chicago Press), 623

Modjaz, M., Kirshner, R. P., Blondin, S., Challis, P., \& Matheson, T. 2008, ApJ, 687, L9

Nordhaus, J., Burrows, A., Almgren, A., \& Bell, J. 2010, ApJ, 720, 694

Park, S., Roming, P. W. A., Hughes, J. P., et al. 2002 ApJ, 564, L39

Reed, J. E., Hester, J. J., Fabian, A. C., \& Winkler, P. F. 1995, ApJ, 440, 706

Rest, A., Foley, R. J., Sinnott, B., et al. 2011, ApJ, 732, 3

Rest, A., Matheson, T., Blondin, S., et al. 2008, ApJ, 680, 1137

Rest, A., Suntzeff, N. B., Olsen, K., et al. 2005, Nature, 438, 1132

Reynoso, E. M., Goss, W. M., Dubner, G. M., Winkler, P. F., \& Schwarz, U. J. 1997, A\&A, 317, 203

Scheck, L., Kifonidis, K., Janka, H., \& Müller, E. 2006, A\&A, 457, 963

Shibata, M., Liu, Y. T., Shapiro, S. L., \& Stephens, B. C. 2006, Phys. Rev. D, 74, 104026

Smith, J. D. T., Rudnick, L., Delaney, T., et al. 2009 ApJ, 693, 713

Stone, R. P. S. 1977, ApJ, 218, 767

Tanaka, M., Kawabata, K. S., Hattori, T., et al. 2012, ApJ, 754, 63

Tananbaum, H. 1999, IAU Circ., 7246, 1

Taubenberger, S., Valenti, S., Benetti, S., et al. 2009, MNRAS, 397,677

Thorstensen, J. R., Fesen, R. A., \& van den Bergh, S. 2001, AJ, 122, 297

Ugliano, M., Janka, H.-T., Marek, A., \& Arcones, A. 2012, ApJ, 757,69

van den Bergh, S., \& Dodd, W. W. 1970, ApJ, 162, 485

van den Bergh, S., \& Kamper, K. W. 1983, ApJ, 268, 129

van den Bergh, S., \& Kamper, K. 1985, ApJ, 293, 537

Vogt, F., \& Dopita, M. A. 2010, ApJ, 721, 597

Vogt, F., \& Dopita, M. A. 2011, Ap\&SS, 331, 521

Wang, L., \& Wheeler, J. C. 2008, ARA\&A, 46, 433

Wang, L., Wheeler, J. C., Höflich, P., et al. 2002, ApJ, 579, 671

Wheeler, J. C., Maund, J. R., \& Couch, S. M. 2008, ApJ, 677, 1091

Wheeler, J. C., Meier, D. L., \& Wilson, J. R. 2002, ApJ, 568, 807

Willingale, R., Bleeker, J. A. M., van der Heyden, K. J., Kaastra, J. S., \& Vink, J. 2002, A\&A, 381, 1039

Winkler, P. F., Twelker, K., Reith, C. N., \& Long, K. S. 2009, ApJ, 692, 1489

Woosley, S. E., Langer, N., \& Weaver, T. A. 1993, ApJ, 411, 823

Woosley, S., \& Janka, T. 2005, Nature Physics, 1, 147

Young, P. A., Fryer, C. L., Hungerford, A., et al. 2006 , ApJ, 640, 891 